\title{
Characterization of Gadolinium Doped Cerium (IV) Oxides Deposited by Reactive Spray Deposition Technology for Intermediate Temperature Fuel Cell Applications
}

\author{
Abhinav Poozhikunnath ${ }^{1}$, Mark Aindow ${ }^{1}$ and Radenka Maric ${ }^{2}$ \\ 1. Materials Science and Engineering Department, University of Connecticut, Storrs CT, USA. \\ 2. Center for Clean Energy Engineering, University of Connecticut, Storrs CT, USA.
}

Intermediate Temperature Solid Oxide Fuel Cells (ITSOFC) operate at lower temperatures $\left(300-700^{\circ} \mathrm{C}\right)$ than traditional SOFCs, and have an improved operational life and reduced running cost due to a reduction in issues related to thermal mismatch, reactivity and thermally activated degradation mechanisms in fuel cell components. Gadolinium Doped Ceria (GDC) is a potential candidate as both electrolyte and electrode material in ITSOFCs, partly due to its high ionic conductivity at $300-700^{\circ} \mathrm{C}$. The performance of GDC electrolyte and electrode layers is critically dependent on the film thickness, morphology and particle size which in turn depend on the film synthesis/deposition technique [1].

The synthesis technique used here is called Reactive Spray Deposition Technology (RSDT). It is a single step, flame-based spray deposition technique for fabricating SOFC components [2]. The deposition process involved atomizing a precursor solution containing dissolved Gadolinium and Cerium precursors $(\mathrm{Gd} / \mathrm{Ce}$ molar ratio of 1/9) and igniting a mixture of the atomized spray and oxygen. The flame provided the heat for precursor decomposition and the transport medium for nanoparticles thus produced. In this study, several $\mathrm{Gd}_{0.1} \mathrm{Ce}_{0.9} \mathrm{O}_{1.95}$ thin films were deposited on $50 \%$ porous Strontium Lanthanum Titanate substrates, by varying either precursor solution concertation or the stand-off distance (defined in fig. 1 (a)). The effect of these deposition parameters on the film thickness, morphology and particle size have been analyzed using a combination of XRD, SEM and TEM.

The phase and composition of the deposited GDC films were analyzed using XRD and EDXS. Average particle size was measured from bright field TEM images, while sharp diffraction rings confirmed crystallinity. Fig. 1 (b)-(d) show the bright field TEM images of selected samples. The particle size was found to decrease with decreasing precursor concentration over a range of $23 \mathrm{~nm}$ to $5 \mathrm{~nm}$. A significant difference in the film morphology was observed when varying the concentration. As shown in the planview SEM images in fig. 2 (a)-(c), the GDC agglomerates appear to be more closely packed, creating a relatively denser film, at lower concentrations. The average film thickness was found to decrease with decreasing concentration because the amount of material being deposited, in the same amount of time, decreased. Increasing the stand-off distance was found to reduce the film thickness almost linearly (fig. 2 (d)-(f)). Differences in the film morphology at different stand-off distances is believed to be due to differences in substrate temperature. The morphology of the GDC film was also dependent on the roughness, porosity and pore size of the substrate. Further investigations into the growth of GDC films on dense substrates and the deposition of a fully dense film are currently ongoing. [3]

References:

[1] C. Xia and M. Liu, Solid State Ionics 152-153 (2002), p. 423.

[2] R. Maric et al, Journal of Power Sources 195 (2010), p. 8198.

[3] The authors acknowledge financial support from ARPA-E (DE-AR0000503). The microscopy studies were performed in the UConn/FEI Center for Advanced Microscopy and Materials Analysis (CAMMA). 


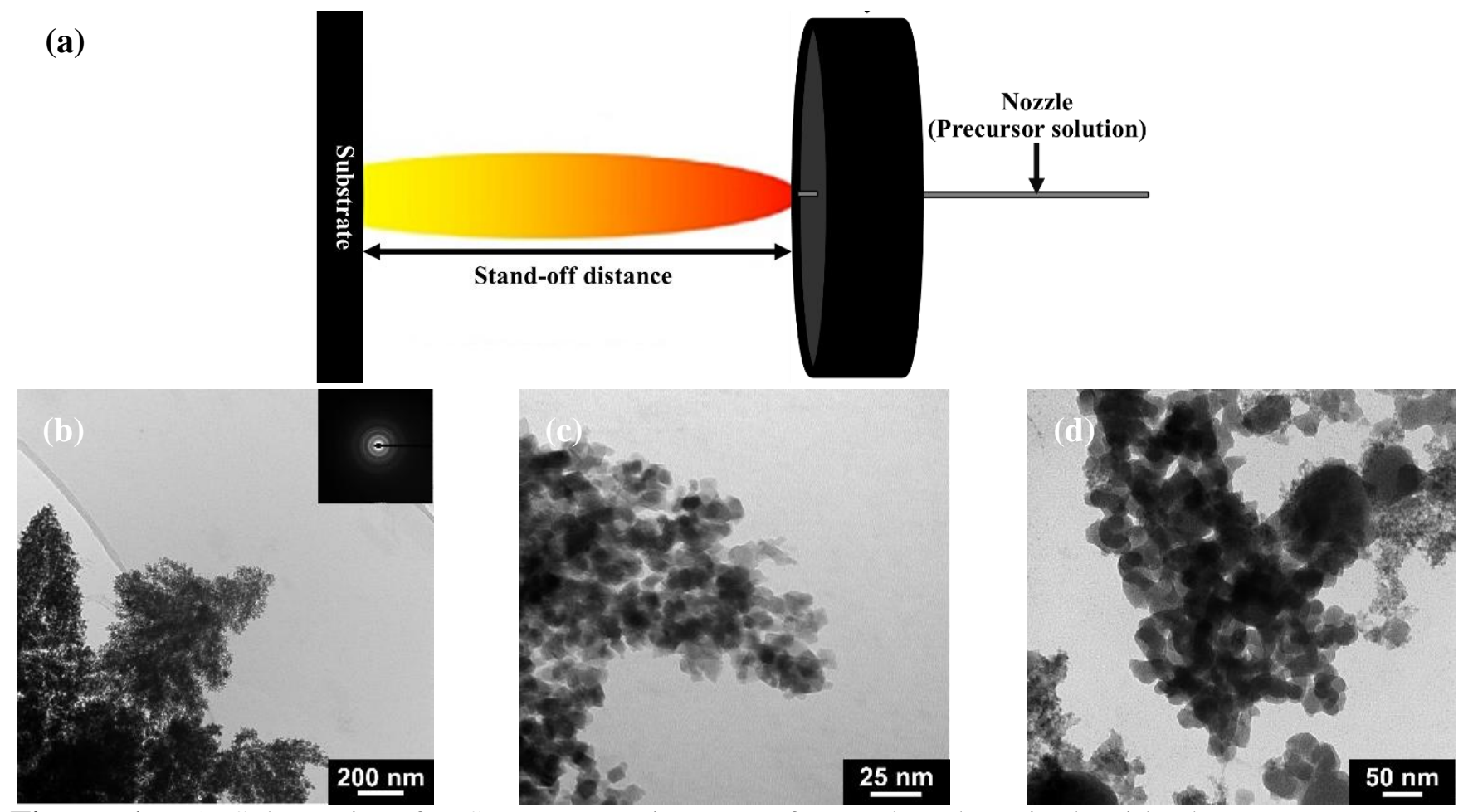

Figure 1. (a) Schematic of RSDT. TEM images of samples deposited with the same precursor concentration but at (b),(c) shorter and (d) longer stand-off distances.
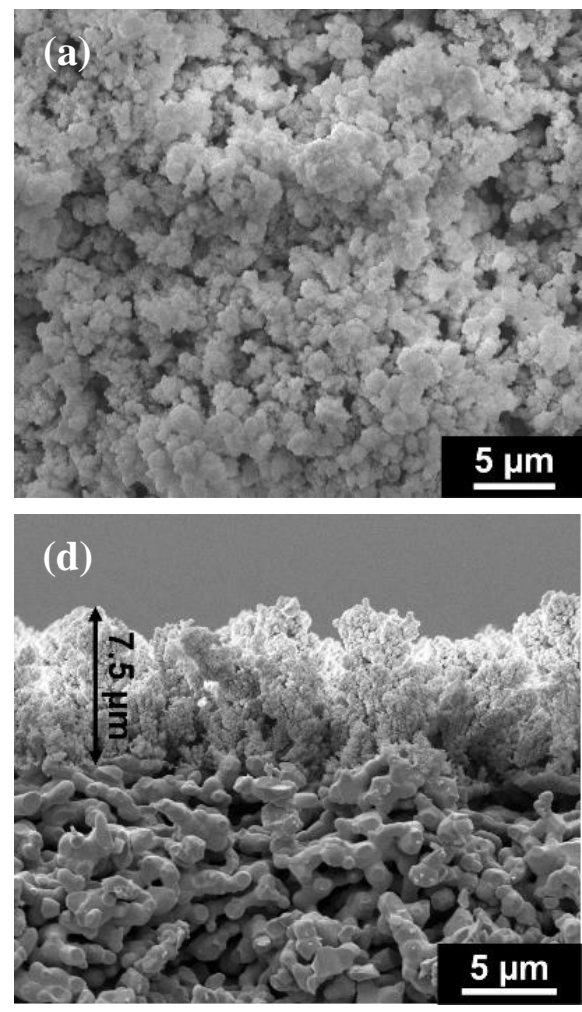
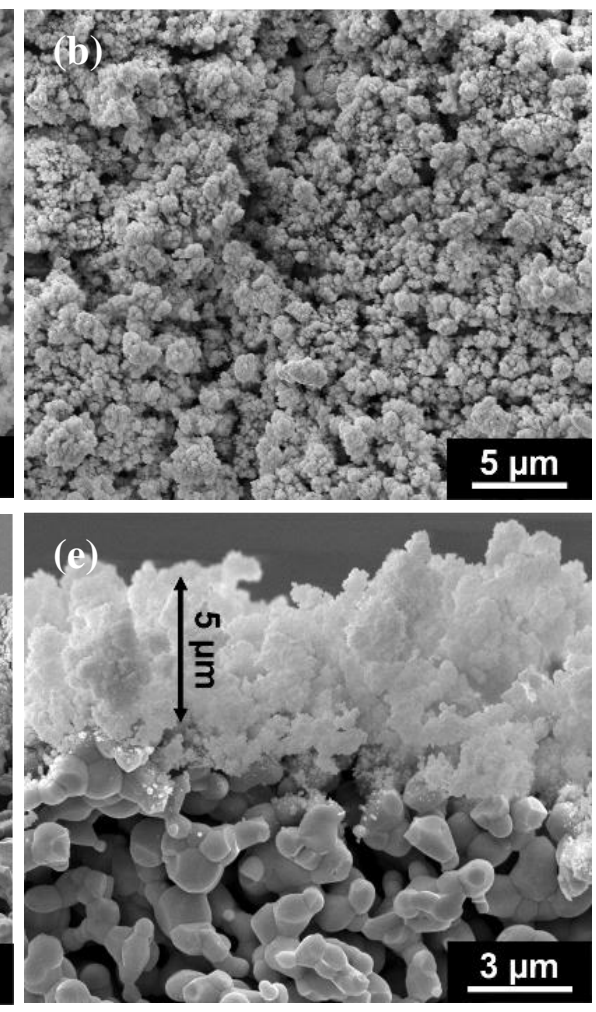
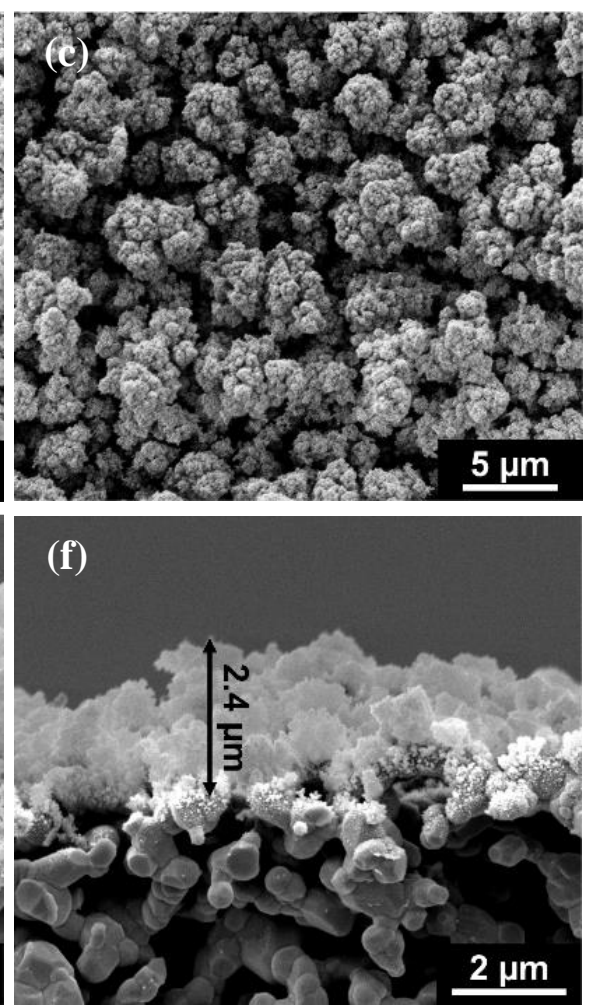

Figure 2. Selected SEM images of deposited GDC films. (a)-(c) plan-view SEM images of samples deposited in increasing concentration, but at same stand-off distances, and, (d)-(f) cross-sectional SEM images of samples deposited with the same precursor concentration, but at increasing stand-off distances. 